\title{
Plattform-Urbanismus
}

Arbeit, Migration und die Transformation des urbanen Raums

\author{
Moritz Altenried \\ Stefania Animento \\ Manuela Bojadžijev
}

\begin{abstract}
Der Beitrag analysiert, wie digitale Plattformen urbanes Arbeiten und Leben ebenso verändern wie die gelebte Räumlichkeit und die materielle Architektur der Stadt. Davon sind nicht nur Arbeitsverhältnisse berührt, sondern auch alltägliche Formen und Praktiken von Mobilität, Konsum oder Reproduktion. Basierend auf umfassenden ethnografischen Forschungen beschreiben wir erstens den Aufstieg der Plattformarbeit in Berlin, insbesondere am Beispiel von Uber, Deliveroo und Helpling. Wir nehmen neue Formen algorithmischer Organisation, Kontrolle und Überwachung von Arbeit im Stadtraum in den Blick und zeigen, dass Plattformarbeit primär migrantisch ist. Davon ausgehend skizzieren wir zweitens die Umrisse eines entstehenden Plattform-Urbanismus. Das umfasst sowohl ein Verständnis der Räume und Geografien digitaler Plattformen als auch eine theoretische Perspektivierung des Begriffs. Drittens betonen wir, dass kritische Analysen des emergenten Plattform-Urbanismus zeigen können, wie Plattformen darauf abzielen, unverzichtbare urbane Infrastrukturen zu werden. Allerdings zeigt sich, dass diese Infrastrukturwerdung urbaner Plattformen kein reibungsloser Prozess ist, sondern politisch und ökonomisch umkämpft.
\end{abstract}

Ersteinreichung: 11. Mai 2020; Veröffentlichung online: 23. April 2021

An English abstract can be found at the end of the document.

\section{Einleitung: Berlin, Juli 2019}

Ein diskreter Klingelton seines Smartphones weist den Fahrradkurier auf einen neuen Auftrag hin. Er bremst am Straßenrand und öffnet die App der Essenslieferplattform Deliveroo. Eine kurze Lieferung aus Berlin-Kreuzberg zum Neuköllner Hermannplatz für eine Entlohnung von Euro 4,20 wird ihm angeboten. Schnell kalkuliert er Entfernung, potenzielle Wartezeiten und die momentane Auftragssituation: könnte sich lohnen. Er akzeptiert den Auftrag mit einem Wisch auf dem Smartphone und macht sich auf den Weg zum Restaurant.

Das Restaurant entpuppt sich als eine unauffällige Küche im Hinterhof eines Kreuzberger Altbaus: ein virtuelles Restaurant, eigentlich nur eine Küche, in der ausschließlich zur Bestellung über verschiedene Essenslieferplattformen gekocht wird. Dementsprechend sind die Abläufe optimiert. Es gibt keine Laufkundschaft, nur die Kurier_innen der verschiedenen Lieferdienste gehen ein und aus. Der Kurier betritt die Küche gemeinsam mit zwei Kolleginnen, die für dieselbe Plattform arbeiten. Er stellt zufrieden fest, dass 
die Bestellung bereits in braunen Pappbehältern auf ihn wartet. Um nicht aus Versehen mit einer falschen Lieferung loszufahren, vergleicht er die Liefernummer in seiner App genau mit der Nummer auf der Papiertüte und markiert die Bestellung - koreanische Burger und eine japanische Bowl - in der App als abgeholt. Eilig packt er die Bestellung in seinem Rucksack und macht sich auf den Weg zum Ziel in Neukölln.

Dort angekommen, bestätigt der Deliveroo-Rider in seiner App die Ankunft bei der Kundschaft und klingelt dann an der Tür. Mehrere mit Zahlencodes gesicherte Boxen unterhalb der Klingeln und Klingelschilder, auf denen statt Namen nur die Nummern der einzelnen Wohnungen stehen, lassen darauf schließen, dass es sich um Ferienwohnungen handelt. Niemand antwortet. Er klingelt erneut. Die beiden jungen Frauen aus London, die sich hier für ein paar Tage ein Appartement über Airbnb gemietet haben, um Berlin zu besuchen, sind noch nicht da. Sie haben das Essen über ihr Smartphone aus dem Uber-Taxi bestellt, dabei jedoch die Dauer der Fahrt zurück zu ihrem Appartement unterschätzt. Die Apps des Lieferdienstes Deliveroo, der Wohnungsplattform Airbnb und des Fahrdienstes Uber befanden sich bereits vor der Berlinreise auf ihrem Handy. All diese Dienste stehen ihnen auch in ihrer Londoner Heimat zur Verfügung - genauso wie in vielen anderen Städten der Welt.

Während der Kurier ungeduldig wartet, öffnet sich die Tür und eine Frau mit Putzsachen tritt heraus. Sie hat die Zeit, während die beiden Londonerinnen beim Sightseeing waren, genutzt, um die Wohnung sauber zu machen. Wie der Deliveroo-Kurier und die Uber-Fahrerin arbeitet auch sie über eine digitale Plattform: Das deutsche Unternehmen Helpling vermittelt unbürokratisch und stundenweise solo-selbstständige Putzkräfte, perfekt für die Besitzer_innen der mindestens fünf Airbnb-Wohnungen in diesem Haus. Diese können so aus der Distanz ihre Wohnungen sauber halten und vermieten. Der Deliveroo-Fahrer nickt der Helpling-Arbeiterin zu und nutzt die Chance, um in den Hausflur zu treten. Er ist selbstständig und wird für die Lieferstrecke bezahlt, nicht für die Zeit, die er für die Auslieferung benötigt. Wartezeiten senken also seinen Stundenlohn. Er überlegt, die Lieferung vor die Wohnungstür zu stellen und dann weiterzufahren, um heute noch sein selbst gestecktes Einkommensziel zu erreichen. Während er noch das Risiko einer verschwundenen Bestellung und den potenziellen Ärger mit der Plattform abwägt, kommen die beiden Londonerinnen zur Tür hinein und freuen sich, dass ihr Mittagessen bereits da ist.

\section{Plattform-Urbanismus}

Diese nur leicht fiktionalisierte Szene aus unserer ethnografischen Forschung vom Sommer 2019 veranschaulicht etwas, das sich als Plattform-Urbanismus bezeichnen lässt. Dies zeigt ein Blick auf die Smartphones von Menschen aus besonders mobilen Bevölkerungsgruppen, wie den beiden erwähnten Touristinnen oder die der meist migrantischen Arbeiter_innen von Plattformen wie Uber, Deliveroo oder Helpling besonders deutlich. Die verschiedenen Apps spielen im Leben dieser oft neuen oder temporären Berliner_innen häufig eine elementare Rolle. Doch auch ein Blick auf das Smartphone einer beliebigen langjährigen Berlinerin wird mit großer 
Wahrscheinlichkeit die Apps vieler solcher Plattformen aufweisen, die das urbane Leben in allen Dimensionen zu durchdringen beginnen. Google Maps leitet die Menschen durch die Stadt und modifiziert dabei ihre Geografie; Amazon beeinflusst den Konsum und die Liefervans verstopfen die Straßen. Praktiken und Räume des Datings und Ausgehens verändern sich durch Apps wie Tinder und Grindr. ShareNow, Lime, und viele andere vermieten über ihre Apps Elektroroller, Fahrräder und Autos und wollen so die urbane Mobilität revolutionieren. Airbnb bietet Ferienappartements, Uber Taxifahrten, TaskRabbit Aufbauhilfen für die neuen Ikea-Möbel, Deliveroo das Mittagessen, Helpling die Reinigung der Wohnung, Care.com die Kinderbetreuung und so weiter. Es gibt kaum noch einen Bereich von Arbeit und Leben, in denen digitale Plattformen keine Rolle spielen. Das datengetriebene Geschäftsmodell der meisten Plattformen basiert darauf, dass sie sich über die Smartphones in den Alltag der Nutzer_innen einflechten und zu einem unverzichtbaren Teil der Infrastruktur im alltäglichen Leben werden. Städte sind das primäre Aktionsfeld dieser digitalen Plattformen und werden so zu einem Laboratorium gesellschaftlicher Veränderung. Mit ihnen verändert sich auch die Stadt selbst und wird, wie die Architektin und Urbanistin Clare Lyster schreibt, immer mehr zu einer „integrierten Serviceplattform“" (Lyster 2016: 13).

Wie digitale Plattformen urbanes Arbeiten und Leben, aber auch gelebte Räumlichkeit und sogar die materielle Architektur der Stadt verändern, ist die zentrale Fragestellung dieses Aufsatzes. Dabei liegt unser Fokus zunächst auf der Analyse von Plattformarbeit in urbanen Räumen (hier konkret in Berlin). Im zweiten Teil des Aufsatzes erweitern wir dann unseren Fokus und fragen, wie Plattformen nicht nur Arbeit, sondern zunehmend auch urbane Räume zu verändern und zu prägen beginnen.

Wir beginnen unsere Analyse mit einer kurzen Eingrenzung des Begriffs Plattform und skizzieren kurz die Geschichte und Relevanz der Plattformarbeit bzw. Gig Economy. Anschließend analysieren wir auf der Grundlage unserer ethnografischen Forschung in Berlin, die ihren Schwerpunkt auf die Plattformen Uber, Deliveroo und Helpling legt, Prozesse und Logik der Appbasierten Plattformarbeit im Stadtraum. Dabei untersuchen wir auch den in der Forschung bislang weitgehend vernachlässigten Aspekt, dass es sich bei dieser Arbeit vorwiegend um migrantische Arbeit handelt. Ausgehend von der Analyse dieses spezifischen Feldes erweitern wir dann unseren Blick auf weitere Fragen des urbanen Lebens, wie die gelebte Geografie oder die urbane Verräumlichung digitaler Plattformen. Zum Schluss erlaubt uns eine kurze Diskussion des Begriffs Plattform-Urbanismus, die gegenwärtigen Transformationen als Infrastrukturwerdung von Plattformen im Urbanen zu charakterisieren. Abschließend wagen wir einen Ausblick auf die Rolle von Plattformen im Kontext der aktuellen Covid-19-Krise.

\section{Forschungsperspektive: Plattformen, Arbeit, Stadt}

Wir verstehen digitale Plattformen sowohl als konkreten und wichtigen Faktor der Transformation urbaner Arbeits- und Lebensverhältnisse, als auch als radikalen Ausdruck breiterer gesellschaftlicher Veränderungen. $\mathrm{Zu}$ diesen gehören die Flexibilisierung von Arbeit, die Logistifizierung von 
Produktion und Alltag sowie das datengestützte und zunehmend automatisierte Management von Arbeit und urbanem Leben. In diesem Text geht es uns nicht um die Behauptung, die Stadt von morgen würde nach dem Prinzip von Uber und Airbnb regiert (auch wenn uns diese These nicht ganz unplausibel erscheint) oder um die Ausarbeitung einer fertigen Groß-Theorie zum Plattform-Urbanismus. Stattdessen nutzen wir den Begriff heuristisch. Wir setzen unsere Forschung zum Einfluss digitaler Plattformen auf städtische Arbeit und städtischen Alltag ein, um eine kritische Perspektive auf die Stadt von heute zu gewinnen. Diese Perspektive auf die digitalisierte Stadt steht aus unserer Sicht im Dialog mit anderen Forschungsperspektiven. Dazu zählen wir etwa kritische Perspektiven auf den Diskurs und die Materialität von Smart Cities oder die Finanzialisierung der Stadt (Halpern et al. 2013; Harvey 2013; Beverungen/Sprenger 2017).

Unsere Analyse digitaler Plattformen im städtischen Raum entwickeln wir primär aus der Perspektive der Arbeit und aus einer Untersuchung von Plattformen, bei denen die Organisation und Ausbeutung lebendiger Arbeit im Zentrum des Geschäftsmodells steht. Unsere Forschung folgt auch deshalb diesen arbeitsvermittelnden Plattformen, weil wir Arbeit und politische Ökonomie für zentrale (wenn auch bei Weitem nicht die einzigen) Faktoren in der Produktion des urbanen Raums halten (Harvey 2010; Briken 2018). Dabei ist uns bewusst, dass wir bei der Analyse von Plattformen im Stadtraum auch andere räumliche Praktiken und Akkumulationsformen in den Vordergrund stellen könnten. Der schillernde Begriff der Plattform umfasst viele verschiedene ökonomische Formationen, die sich teilweise sehr stark von den hier untersuchten Plattformen wie Uber oder Deliveroo unterscheiden (Gillespie 2010; Srnicek 2016). Deren Einfluss und deren Rolle im urbanen Raum ist wiederum jeweils sehr unterschiedlich - man denke an die sehr unterschiedlichen Auswirkungen auf das urbane Leben durch die bereits genannten Apps Grindr und Tinder (Miles 2017), Google Maps (LuqueAyala/Neves Maia 2019), Airbnb (Wachsmuth/Weisler 2018) oder städtische Datenplattformen (Barns 2018). Diese sehr willkürliche Auswahl zeigt auch, dass die Subsumption unterschiedlicher Applikationen und Unternehmen unter den Begriff der Plattform (und des Plattform-Urbanismus) zwar zu dessen Attraktivität, aber auch zu dessen Problematik beiträgt. Schließlich erlaubt es der Begriff, sehr unterschiedliche Unternehmen und Konstellationen zu vereinheitlichen und droht dadurch, an analytischer Schärfe zu verlieren. Andererseits erlauben Begriffe wie Plattform und Plattform-Urbanismus eine breitere Perspektive, die sehr hilfreich dabei sein kann, die tiefgreifenden Transformationsprozesse zu begreifen, zu deren Resultaten etwa die ungeheure globale Macht von Unternehmen wie Amazon oder Facebook gehört. Die Frage, welche Logik diese unterschiedlichen Unternehmen miteinander verbindet, lassen wir im Folgenden jedoch weitgehend offen. Stattdessen entwickeln wir unseren Beitrag primär aus der Perspektive der auch als Gig Economy bezeichneten Plattformarbeit. Damit konzentrieren wir uns auf einen bestimmten Typus von Plattformen sowie auf einen spezifischen Aspekt der Plattformisierung des Urbanen.

Wir entwickeln unsere Argumentation vor dem Hintergrund zweier laufender Forschungsprojekte zu Plattformarbeit, Stadt und Migration.[1] Diese Projekte verbinden Ansätze und Perspektiven aus Anthropologie, 
Sozialwissenschaften, Migrationsforschung, Geografie und Stadtforschung sowie Politischer Ökonomie. Methodisch verfolgen wir schwerpunktmäßig einen qualitativen und ethnografischen Forschungsansatz. Die primäre Grundlage für diesen Artikel liefert uns unsere empirische Forschung in Berlin. Dazu gehören umfangreiche ethnografische Untersuchungen (onund offline), über 40 Interviews mit Plattformarbeiter_innen von Deliveroo, Helpling und Uber sowie Interviews mit Gewerkschafter_innen, Aktivist_innen, Verwaltungsmitarbeiter_innen, Abgeordneten und weiteren Akteur_innen. Diese Forschung in Berlin steht im Kontext eines zweiten, transnationalen Forschungsprojektes zu Plattformarbeit in verschiedenen europäischen Städten, das uns die Kontextualisierung der besonderen Berliner Situation erlaubt.

\section{Plattformarbeit in der digitalisierten Stadt}

„I was told that it was an application and that I was going to get a job right away. They told me that you register and you get offers through the application, it was strange to me, but it was like that, you register and you get offers, cleaning offers from different houses, from different areas, either two hours or four hours, but Helpling gets a pretty high commission. That's basically it." Camila, Berliner Plattformarbeiterin aus Argentinien (Interview, Januar 2020)[2]

Plattformarbeit ist heute ein globales Phänomen. Millionen Menschen arbeiten in urbanen Zentren auf der ganzen Welt über digitale Plattformen. „Everybody is talking about the gig economy“, schreiben die britischen Wissenschaftler Jamie Woodcock und Mark Graham in ihrer kritischen Einführung zum Thema (Woodcock/Graham 2019: 1). Während sich der Begriff in Deutschland eher langsam verbreitet, hat „Gig Economy“ in anderen Ländern bereits Einzug in die Umgangssprache gehalten. In Großbritannien etwa, wo Woodcock und Graham zufolge die Zahl der Plattformarbeiter_innen inzwischen der Zahl der Beschäftigten im öffentlichen Gesundheitssektor entspricht, entwickeln sich seit einigen Jahren breite öffentliche Diskussionen über das Phänomen und dessen Auswirkungen auf die Welt der Arbeit. Die Aufmerksamkeit für die Gig Economy speist sich hier, aber auch anderswo in Europa, nicht zuletzt aus einer Welle von Protesten von Plattformarbeiter_innen (Animento et al. 2017; Cant 2019). Vermittelt durch diese Kämpfe gewinnt der Begriff inzwischen auch in der deutschsprachigen Debatte an Bedeutung, vor allem im Bereich der Essenslieferung.

Zur Größe der Gig Economy gibt es wenig belastbare Zahlen und kontroverse Diskussionen. Dies liegt nicht zuletzt daran, dass Plattformarbeit oft aus dem Raster üblicher Erhebungsmethoden zu Arbeitsmärkten fällt, teilweise nicht statistisch erfasst wird bzw. werden kann, häufig ein Zweitoder Drittjob ist und zudem nur schwer von ähnlichen Formen flexibler und kontingenter Arbeit abgrenzbar ist. Die Plattform Helpling etwa, deren Geschäftsmodell das Eingangszitat prägnant beschreibt, vermittelt in über 200 Städten weltweit Putzkräfte. Die selbstständigen Kuriere von Deliveroo liefern Essen in über 500 Städten aus und die Taxiplattform Uber hat über 100 Millionen Kund_innen in über 900 Städten auf der ganzen Welt. Grob 
geschätzt dürften es zusammen mit Tausenden weiterer Plattformen insgesamt an die 50 Millionen Plattformarbeiter_innen sein (Heeks 2019).

Eine umfangreiche Studie in 13 europäischen Ländern, die den BegriffGig Economy relativ breit definiert und auch digitale Arbeit auf Onlineplattformen wie Amazon Mechanical Turk miteinschließt, resümiert, die Plattformarbeit spiele in allen untersuchten Ländern eine bedeutende Rolle. Allerdings sei sie in den meisten Fällen nicht die Haupteinnahmequelle, sondern eine wichtige Ergänzung anderer Einkommensquellen. Interessant ist die geografische Verteilung: Die höchsten Anteile regelmäßiger (wöchentlicher) Plattformarbeit wurden in Zentral- und Osteuropa gemessen. So waren es in Tschechien 2019 etwa 28,5 Prozent der arbeitenden Bevölkerung, während die Werte in Nord- und Westeuropa geringer sind. In Deutschland gingen der Studie zufolge 2016 etwa 6,2 Prozent der arbeitenden Bevölkerung mindestens einmal wöchentlich unterschiedlichen Formen von Plattformarbeit nach (Huws et al. 2019). Eine Untersuchung der Europäischen Kommission kommt mit einer anderen Methode auf ähnliche Zahlen für Deutschland (Pesole et al. 2018). Andere Erhebungen kommen zu etwas niedrigeren Zahlen. Plattformarbeit macht also bisher - darauf deuten die vorliegenden Zahlen hin - einen eher kleinen Anteil an den jeweiligen nationalen Arbeitsmärkten aus. Dieser Anteil scheint jedoch stark zu wachsen. Der Studie von Ursula Huws (2019: 1) zufolge hat sich etwa die Zahl der Plattformarbeiter_innen in Großbritannien zwischen 2016 und 2019 verdoppelt.

Die Gig Economy ist ein globaler Arbeitsmarkt mit primär urbanen Effekten. Daher ist seine genaue Größe in einzelnen nationalen Ökonomien vielleicht auch nicht der springende Punkt. Ihre qualitative Relevanz erhält sie zumindest auch durch ihre Eigenschaft als zentrales Experimentierfeld für neue Formen digital vermittelter, organisierter und kontrollierter Arbeit. So deutet vieles darauf hin, dass die Covid-19-Krise und die daraus hervorgehenden, schon vorauszusehenden wirtschaftlichen Verwerfungen die Bedeutung der Plattformarbeit noch einmal verstärken werden. Bereits in ihrer heutigen Form ist die Gig Economy ein Produkt der letzten großen Finanz- und Schuldenkrise. Zwar steht die Gig Economy historisch betrachtet in einer langen Genealogie kontingenter Arbeit, sie ist aber in ihrer heutigen Form ein Produkt der globalen Krise ab 2007. In dieser trafen insbesondere in den USA Arbeitskräfte, die aufgrund von Entlassungen und Rezession freigesetzt worden waren, auf großzügig mit Risikokapital ausgestattete Plattformunternehmen und begründeten so einen neuen Kreislauf kontingenter Arbeit.

Die 2009 gegründete Taxi-Plattform Uber lieferte dafür eine Art Blaupause: Wie die meisten Plattformen versteht sich Uber primär als Technologieunternehmen, das zwischen Kund_innen und selbstständigen Taxiunternehmer_innen vermittelt. Dementsprechend hat das Unternehmen selbst nur wenige festangestellte Mitarbeiterinnen. Es organisiert eine große Menge Arbeitskraft on demand, um flexibel auf sie zugreifen zu können. Den etwa 20.000 fest angestellten Mitarbeiter_innen bei Uber stehen weltweit über drei Millionen formell selbstständige Fahrer_innen gegenüber. Diese führen mit ihren eigenen Autos einzelne Taxifahrten durch, die ihnen über die Uber-App vermittelt und durch diese einzeln abgerechnet werden. Dieses Modell - die Auslagerung einzelner, zeitlich befristeter Aufträge (der sogenannten Gigs) an formell selbstständige Arbeiter_innen mittels 
einer digitalen Plattform - bildet die Grundlage der heutigen Gig Economy. Inzwischen dringen solche Plattformmodelle in fast alle Bereiche vor.

Uber verbindet sein schlankes Geschäftsmodell (Srnicek 2016), das Fixkosten für Arbeit und Autos weitgehend vermeidet, mit einer kapitalintensiven Disruptionsstrategie. Diese zielt darauf ab, herkömmliche Taxidienste zu unterbieten und so vom Markt zu verdrängen. Zudem lässt sich eine oftmals aggressive Strategie zur Umgehung nationaler und munizipaler Gesetze und Reglungen im Bereich des Personentransports beobachten. Diese führt vielerorts - wie aktuell auch in Deutschland - zu erbitterten Konfrontationen mit Taxiverbänden und Stadtregierungen. Diese Auseinandersetzungen sorgen immer wieder für Rückschläge in der globalen Expansionsstrategie von Uber. Auch das Modell der solo-selbstständigen Fahrer_innen ist rechtlich wie politisch an vielen Orten umkämpft.

\section{Plattformarbeit in Berlin: Kontingenz und Kontrolle}

Auch in Berlin ist das Geschäft von Uber rechtlich und politisch umstritten. Ein Effekt dieser Auseinandersetzungen ist, dass die Beschäftigungsverhältnisse bei Uber hier heterogener ausfallen als an anderen Standorten. In Berlin stehen zwischen Plattform und Fahrer_in oft noch verschiedene Subunternehmen. Zugleich ist Uber für viele Fahrer_innen tatsächlich ein Vollzeitjob, auf dessen Einkünfte sie nicht verzichten können (für die Situation in den USA und Kanada: Rosenblat 2018). Verschärft wird diese Situation oft durch Schulden oder Leasinggebühren, mit denen die Fahrer_innen die Autos für ihre Arbeit finanziert haben und die sie regelmäßig bedienen müssen - nicht selten bei Uber selbst. Petra, eine Berliner Uber-Fahrerin, die mit dieser Tätigkeit ihre Rente aufbessert, beschreibt die Situation so: „Die LeasingAutos, die müssen ja immer bezahlt werden. Da ist das Leasing, die Versicherung und das Geld muss natürlich irgendwie reinkommen. Deshalb darf ein Auto auch nicht stillstehen." (Interview März, 2020) Petra erzählt, dass sich manchmal zwei Fahrer_innen ein Auto teilen, um es 24 Stunden am Tag zu nutzen und so rentabel zu machen. Ihr Arbeitsalltag wird dabei komplett von der Uber-App auf ihrem Smartphone organisiert. Durch diese bekommt sie ihre Aufträge für die einzelnen Fahrten, die Routen und eine Reihe weitere Informationen. Mit den angestellten Mitarbeiter_innen von Uber im Berliner Büro hat Petra nur in Ausnahmefällen zu tun.

Wie eingangs beschrieben, werden auch die Fahrer_innen der Essenslieferplattform Deliveroo per App durch die Stadt dirigiert. Der hohe Grad an Kontrolle über den Arbeitsprozess, den viele Plattformen über ihre Apps ausüben, lässt die Argumentation der Plattformen, lediglich Aufträge für Selbstständige zu vermitteln, zumindest fragwürdig erscheinen. Komplexe Algorithmen verteilen die Aufträge an die Fahrer_innen und versuchen diese mit Anreizsystemen in Zonen mit hoher Nachfrage zu locken. Gleichzeitig dient die App zur Schichtplanung und zur automatisierten kleinteiligen Organisation jeder einzelnen Lieferung oder Taxifahrt. Dabei werden verschiedenste Daten erhoben wie Fehlzeiten, Verspätungen, Geschwindigkeit, Routen, abgelehnte Aufträge oder Bewertungen der Kundschaft. Diese Daten werden sowohl genutzt, um die Algorithmen zu optimieren als auch um die Arbeit der einzelnen Fahrer_innen umfassend zu vermessen und gegebenenfalls zu sanktionieren. 
Teil dieses integrativen Systems ,algorithmischen Managements“ (Beverungen 2017) sind verschiedene Bewertungssysteme. Oft strukturieren diese den Zugang zu zukünftigen Aufträgen und dienen so als Disziplinierungsmechanismen für die formell selbstständigen Plattformarbeiter_innen. Bei Uber und Helpling sind darüber hinaus die Bewertungen der Kundschaft enorm wichtig für den Zugang zu zukünftigen Aufträgen. Sie bringen oftmals die Arbeiter_innen in schwierige Situationen gegenüber ihren Kund_innen, etwa wenn diese unbezahlte zusätzliche Leistungen verlangen oder sich übergriffig verhalten. Bei Deliveroo bilden vor allem die individuellen Statistiken zu Pünktlichkeit und Zuverlässigkeit sowie die Bereitschaft, abends und am Wochenende zu arbeiten, die Grundlage für den Zugang zu neuen Schichten und Aufträgen. Chris, ein US-amerikanischer IT-Arbeiter, der auf der Suche nach einem Job in Berlin bei Deliveroo gelandet ist, erklärt: „When you want shifts, it's annoying if you have bad statistics." (Interview, August 2019) Im Falle von kurzfristigen Terminen, Krankheit oder schlechtem Wetter steht Chris vor der Wahl, entweder trotzdem zu arbeiten oder in der folgenden Woche seinen Zugang zu Aufträgen zu gefährden: „A couple of weeks ago it was [...] really bad. I was working, and it was really raining, really hard and it was kind of like a storm. And it wasn't even safe to ride. So I went home early and it still affected my statistics." (ebd.) Sowohl das Zurückgreifen auf die Kundschaft als ,Ko-Management ‘ als auch das Management über Apps und Algorithmen zielen auf ein System, das weitgehend automatisiert funktioniert und daher kaum angestelltes Personal für Betreuung und Management von Arbeiter_innen und Kundschaft verlangt. Digitale Technologie erlaubt dabei die präzise Organisation, Kontrolle und Vermessung der Arbeit der im Stadtraum verteilten Fahrradkurier_innen, Taxifahrer_innen und Paketlieferant_innen in einer Weise, die vorher nur in der disziplinären Architektur einer Fabrik denkbar war. Nun erfolgt sie aus der Ferne und weitgehend automatisiert - die Plattform als „digitale Fabrik“ (Altenried i. E).

Das generelle Ziel von Plattformunternehmen ist es, ihre Fixkosten (für Arbeit und Produktionsmittel) möglichst weit zu senken. Der Rückgriff auf solo-selbstständige Arbeiter_innen, die mit ihren eigenen Fahrrädern oder Autos unterwegs sind, führt im Zuge der Digitalisierung der Arbeitsorganisation zur Renaissance einer eigentlich als weitgehend historisch betrachteten Lohnform: dem Stücklohn. In der Geschichte des Kapitalismus an den Rand gedrängt, wenn auch nie ausgestorben, sind Stücklöhne in der heutigen Gig Economy ein zentrales Mittel, das einerseits unternehmerische Risiken auf die Arbeiter_innen abwälzt und andererseits deren Leistungskontrolle und Disziplinierung dient. Die Arbeiter_innen werden nur für die einzelnen Aufträge bezahlt, nicht für eine festgesetzte Arbeitszeit. So verursachen sie bei Auftragsflauten keinerlei Kosten für die Unternehmen. Gleichzeitig werden auch die Kosten für Schichtplanung und Arbeitswege auf die Arbeiter_innen abgewälzt. Dies ist zum Beispiel ein Problem für Helpling-Arbeiter_innen, deren Arbeitsorte in der ganzen Stadt verteilt sein können, wie die bereits zitierte Camila erklärt: „A lot of time is lost between transfers, in the train, in the bus [...] Today from seven in the morning to three in the afternoon, I didn't stop. And at the end of the day, the second house for two hours paid me 15 euros, and the first one, I think, was 19." (Interview, Januar 2020) 
Stücklöhne funktionieren zugleich als Disziplinierungsinstrument und können so die direkte Kontrolle über den Arbeitsprozess ersetzen. So wirkt sich etwa die Geschwindigkeit eines Deliveroo-Kuriers direkt auf seinen Stundenlohn aus. Die Stücklöhne sind meist flexibel, das heißt sie ändern sich häufig, werden vielfach in Echtzeit an Nachfrage und verfügbare Arbeiter_innen angepasst. Bei Deliveroo ist die Distanz zwischen Abholort und Lieferziel ein zentraler Faktor der Entlohnung. In der Folge müssen die Fahrer_innen jedes Mal, wenn ihnen in der App ein Auftrag angeboten wird, mehrere Faktoren (generelle Nachfrage, potenzielle Wartezeit im Restaurant, Schwierigkeit des Anfahrtsweges, Endpunkt der Lieferung etc.) abwägen, um zu entscheiden, ob es sich für sie lohnt, den Auftrag anzunehmen. Jeder einzelne Auftrag ist stets eine kleine Wette darauf, dass es sich lohnen wird.

Die Plattform Helpling erlaubt es den darauf angemeldeten Putzkräften, ihren Stundenlohn innerhalb einer vorgegebenen Spanne selbst festzulegen. Von diesem Lohn zieht Helpling dann etwa 30 Prozent Vermittlungsgebühr ab. Dadurch stehen die Arbeiter_innen der Plattform in direkter Konkurrenz zueinander um die begrenzten Putzaufträge. Insbesondere Arbeiter_innen mit wenigen oder schlechteren Kund_innenbewertungen können es sich nicht erlauben, ihre Stundenlöhne zu hoch anzusetzen, da sie sonst keine Aufträge mehr erhalten und im Niemandsland der Plattform verschwinden. Natalia, eine weitere Helpling-Arbeiterin aus Argentinien, beschreibt diese Problematik so: „I started to raise [the price] because it doesn't give me enough money to survive, actually. So, I started to upgrade. And then there was a time when it was too high, and I didn't get any offers, so I lowered it once again. Now I have a new model: first more clients, and then I upgrade again.“ (Interview, Januar 2020) Die Gestaltung des Profilfotos, der Klang von Namen und vergeschlechtlichte Zuordnungen sind weitere Faktoren, die bei dieser Konkurrenz eine Rolle spielen.

Die Konkurrenz um volatile Aufträge ist in der Plattformökonomie ein globales Problem. Da selbstständige Arbeiter_innen kaum Fixkosten verursachen, gibt es für die Plattformen selbst kaum Anreize, die Zahl der bei ihnen angemeldeten Arbeiter_innen zu begrenzen, ganz im Gegenteil: Eine hohe Zahl an Arbeiter_innen bietet Plattformen wie Uber und Deliveroo die Möglichkeit, im ganzen Stadtgebiet schnellen Service anzubieten, wie Tommaso, ein Berliner Deliveroo-Fahrer analysiert: „So, their ideal condition would be to have a lot of workers, and then couriers doing just one delivery per hour, and the rest of the time doing nothing." (Interview, Juli 2019). Tommaso ist klar, dass sich seine Auftragslage aufgrund einer Zunahme an latenten Arbeitskräften jederzeit verschlechtern kann. In den meisten Ländern ist das Angebot an Arbeiter_innen größer als die Zahl der vorhandenen Aufträge. Plattformen sind meist nicht gewillt, den Arbeiter_innen eine bestimmte Anzahl an Aufträgen zuzusichern. Um der volatilen Auftragslage besser begegnen zu können, sind viele Arbeiter_innen bei mehreren Plattformen gleichzeitig angemeldet. Sie versuchen so, ein ausreichendes Einkommen zusammenzubringen. Selbst wenn die Auftragslage, wie bei Tommaso gut ist, ist das noch keine Garantie für die Zukunft. Der Fahrer reflektiert darüber:

„The problem was actually, there was no guarantee actually for, no protection as a worker, let's say. So, also there was no guarantee actually, 
the company would keep the same level of average of deliveries per hour. And there was no control actually over how many people were employed. And basically, I could be fired for overnight. The same thing, flexibility, also in their case, so like the dark side of flexibility.“ (Interview, Juli 2019)

Im selben Interview betont der 38-jährige Fahrer aber auch die „hellen“Seiten der Flexibilität. Seit er vor über zehn Jahren aus Norditalien nach Berlin gekommen ist, arbeitet er als selbstständiger Stadtführer. Seine vier Jahre bei Deliveroo erlaubten ihm ein gutes Zusatzeinkommen, besonders im Winter, wenn weniger Besucher_innen nach Berlin kommen. WieTommaso kritisieren viele Plattformarbeiter_innen die Prekarität dieser Existenzweise. Gleichzeitig betonen sie aber, dass diese Arbeit auch ihnen eine flexible Arbeitszeitplanung ermöglicht. Digital organisierte Plattformarbeit funktioniert in diesem Sinne für manche auch als Ergänzung zu anderen Einkommen oder als Zwischenlösung. Doch niemand möchte langfristig oder ausschließlich davon abhängig sein. Während für den italienischen Stadtführer Deliveroo als Zusatzeinkommen funktioniert hat, haben viele andere Plattformarbeiter_innen häufig weniger Alternativen und sind vollständig auf bestimmte Plattformen angewiesen.

Krankheit oder Unfälle stellen eine zusätzliche ständige Bedrohung dieser prekären Kalkulation dar. In den meisten Fällen bedeuten sie einen hundertprozentigen Verdienstausfall. Dem stehen in der Regel kaum Ersparnisse gegenüber. Joaquín, ein Helpling-Arbeiter aus Chile, erläutert: „I would not like to get sick because you practically lose money, you cannot work, there is no security. It is a problem to get sick and it is a problem that you have to solve." (Interview, Dezember 2019) Arbeitsunfälle stellen dabei ein besonderes Problem dar, da viele der Arbeiter_innen nur unzureichend versichert sind. Deswegen versuchen sie, Arbeitsunfälle als private Unfälle darzustellen oder sie suchen bewusst keine medizinische Hilfe, wie Sofía, eine spanische Deliveroo-Fahrerin, schildert:

"I had a crash in November, a pretty big bad crash. I didn't work for like three weeks. And I have like the European [health insurance] card, but yeah, of course, you have like nothing covered. I ride fixed, and I was going down a hill, and the chain came out of place. So yeah, it was pretty bad. And I was like: ,I'm not calling an ambulance.““(Interview, August 2019)

Während einige Arbeiter_innen bewusst daraufverzichten, sich zu versichern, weil dies ihre Einkünfte schmälern würde, liegt es bei vielen häufig an mangelnden Kenntnissen der entsprechenden Gesetze und Regularien sowie ihrer eigenen Rechte. Dies gilt insbesondere für migrantische Arbeiter_innen, für die das deutsche Sozialsystem sowie gesetzliche Regelungen zu Anmeldung, Selbstständigkeit und Versicherung oft auch sprachlich kaum zugänglich sind.

\section{Migration: Plattform-Mobilitäten}

„But at the same time, this is the only option that the immigrants or people from Chile or people from India have. Like they cannot work anywhere else. So, even though the work conditions are shit, they - it's 
the only thing people have, yeah, the only opportunity. So, for me, I was really happy with it. And as long as I didn't get hit by a car, everything was going to be okay." Bastián, Deliveroo-Arbeiter aus Chile (Interview, August 2019)

Plattformarbeit ist vorwiegend migrantische Arbeit. In unseren vorherigen Arbeiten haben wir stets betont, dass Arbeit grundsätzlich nicht ohne die sie konstituierende Mobilität gedacht werden kann (Altenried et al. 2017, Bojadžijev 2020). Dennoch ist die Verbindung zwischen Arbeit und Mobilität historisch unterschiedlich und muss folglich immer wieder neu bestimmt werden. Unsere aktuelle Untersuchung zeigt, dass sowohl in Berlin als auch in den meisten anderen europäischen Städten die deutliche Mehrheit der auf den verschiedenen Plattformen Arbeitenden (oft relativ frisch) zugezogen sind. Während dies bei Uber in Berlin viel mit der langen Migrationsgeschichte des Taxigewerbes in Deutschland zu tun hat, arbeiten bei Deliveroo und Helpling insbesondere junge Migrant_innen, die erst vor Kurzem in die Stadt gezogen sind, etwa aus Lateinamerika oder Südeuropa.

Für vielejüngere Migrant_innen ist Plattformarbeit eine Möglichkeit, direkt nach der Ankunft in Berlin Geld zu verdienen. Die Plattformen verlangen nur ein Minimum an Papieren und da angemeldete Arbeiter_innen keine Fixkosten verursachen, nehmen Plattformen oft alle, die sich bewerben - weitgehend ohne Bewerbungsverfahren, meist ohne Qualifikationsanforderungen und ohne Anlernphase. Die größte Hürde, an andere Jobs zu kommen, sind für die meisten dieser Migrant_innen ihre fehlenden Deutschkenntnisse. Ohne diese verringert sich die Auswahl an verfügbaren Arbeitsmöglichkeiten deutlich; ohne gute Englischkenntnisse sind sie noch weiter eingeschränkt. Die Apps der Plattformunternehmen sind dagegen meistens mehrsprachig und erlauben so auch einen Arbeitseinstieg ohne englische oder deutsche Sprachkenntnisse.

Insbesondere für junge Migrant_innen aus Südamerika, die meist mit einjährigen Visa einreisen, ist die Plattformarbeit eine gute Möglichkeit, den eigenen Lebensunterhalt „in der Migration“ zu verdienen. Für manche wird diese Arbeit zu einem eingeplanten Teil ihrer Migrationsprojekte und stellt so etwas wie eine „Migrationsinfrastruktur“(Xiang/Lindquist 2014) für mobile Arbeiter_innen dar. Bastián, der bereits zitierte junge Chilene, antwortet auf die Frage, inwieweit Plattformen seine Entscheidung nach Berlin zu kommen, beeinflusst haben:

„It's quite known that both Helpling and Deliveroo are the easy jobs to apply to when you come with a visa, because you only have one year, and this is very immediately. You don't need that much papers, and you don't need to speak German. So, yeah, I always thought that it was an option working as Deliveroo, even when I was in Chile." (Interview, August 2019)

Viele migrantische Berliner Plattformarbeiter_innen berichten, dass die Möglichkeit, über Plattformen einen schnellen Einstieg in den Arbeitsmarkt zu finden, ihnen bei ihren Migrationsplänen Zuversicht gegeben hat. Hinzu kommt, dass einige bereits in ihren Heimatländern für Plattformen gearbeitet haben, teilweise sogar für dieselben wie in Berlin. Sie wissen also bereits, wie diese funktionieren. Manche von ihnen haben mittlerweile sogar in mehreren 
Ländern für unterschiedliche Plattformen gearbeitet und organisieren so ihre transkontinentale Mobilität.

Nicht wenige dieser migrantischen Plattformarbeiter_innen haben Universitätsabschlüsse und hoffen darauf, im Laufe der Zeit Arbeit in dem Bereich zu finden, in dem sie ausgebildet wurden. Gustavo, ein Stadtplaner aus Peru, der bei Helpling arbeitet, schildert dies so: „While I look for work in my master's degree, to survive I am working, because, really, if you are fast, you earn more than in a café or restaurant.“ (Interview, Januar 2020) So wie Gustavo nennen die meisten jüngeren Plattformarbeiter_innen Jobs in Cafés oder Restaurants als mögliche Alternative zur Plattformarbeit. Die dortigen Arrangements sind meist ähnlich (schlecht) bezahlt und oftmals noch informeller und prekärer. Deswegen erscheint die per App vermittelte Arbeit ihnen oft als die bessere Option.

Die wenigen Alternativen für migrantische Arbeiter_innen kontextualisieren deren ambivalente Bewertung von Plattformarbeit in den stratifizierten urbanen Arbeitsmärkten. Viele Sektoren, in denen Plattformen in den letzten Jahren eine Rolle zu spielen begannen - insbesondere im Dienstleistungsbereich -, sind historisch von flexibilisierten Arbeitsverhältnissen geprägt. In ihnen sind überdurchschnittlich häufig migrantische und weibliche Arbeiter_innen tätig. In ihrer Analyse der „Global City“ London, diagnostizieren Jane Wills und ihre Kolleg_innen eine Verstärkung, aber auch eine neue Qualität dieser Tendenz. Sie sprechen von einer „neuen migrantischen Arbeitsteilung" und bezeichnen damit eine Stratifizierung des Arbeitsmarktes, bei der sich Migrant_innen vorwiegend am unteren Ende finden: „While migrants have long populated the lower echelons of the London labour market, supplying the workers who do the dirty, dangerous and difficult jobs, we posit that something new has been going on over the past two decades or so. Most clearly in relation to its rise to global-city status, London has become almost wholly reliant on foreign-born workers to do the city's ,bottom-end' jobs.“ (Wills et al. 2010: 1). In jüngerer Zeit spielen in dieser Dynamik digitale Plattformen eine wichtige Rolle. Nicht von ungefähr wurde Deliveroo 2013 in London gegründet. Aus der Perspektive nationaler Arbeitsmärkte mögen die Arbeitsverhältnisse bei digitalen Plattformen oft irregulär erscheinen. Aus der Perspektive migrantischer Arbeit sind sie jedoch Teil einer langen Geschichte hyper-flexibler, prekärer und durch Überausbeutung gekennzeichneter Arbeitsverhältnisse für mobile Bevölkerungen.

Gerade für Berlin - kontinuierliches Ziel verschiedenster Migrationsbewegungen - kann die Rolle migrantischer Arbeit nicht nur im Bereich digitaler Plattformen nur schwer überschätzt werden. Plattformen profitieren von den jungen, oft hochqualifizierten migrantischen Arbeiter_innen und nehmen deren heterogene Mobilitätspraktiken auf. Auch wenn die individuellen Geschichten und Motive der Migration sehr verschieden sind, zeigt sich in vielen Städten ein ähnliches Bild wie in Berlin: Es sind Migrant_innen, die den Großteil der On-Demand-Arbeiter_innenschaft der Plattformen stellen. Das gilt auch über Europa hinaus. Auf die Frage, wie die Arbeit bei Essenslieferplattformen in seiner Heimatstadt Santiago de Chile funktioniert, analysiert Tomás, ein Berliner Deliveroo-Fahrer, eine ähnliche Form der migrantischen Arbeitsteilung: 
„You see in Chile, it has the same effect as here. A lot of people from Colombia and Venezuela are working there. So, it's like a job that is accessible to them. It's just like the same with us, like another country that is coming with difficulties in terms of economics and opportunities." (Interview, Juli 2019)

Die von uns untersuchte Plattformarbeit in Berlin bietet als eine Art Mikrokosmos Einblicke in globale ökonomische und politische Krisen der jüngeren Vergangenheit. Von den Plattformarbeiter_innen kann man ebenso Geschichten über den Krieg in Syrien oder die Währungskrise in Argentinien hören wie über die Verwerfungen der Eurokrise und der Austeritätspolitik in Südeuropa. So berichtet etwa die 22-jährigen Gabriela, dass sie sich in Berlin mehr Chancen auf dem Arbeitsmarkt erhofft als in ihrer Heimat Barcelona:

„To work in Spain is not good. You don't get more than 10 euros per hour. You don't get more. I was working in a restaurant by black. But I was working as dishwasher. But there was also the chef and the waitress. And we all win 5.50 euro per hour, we all." (Interview, Mai 2019)

Gabriela arbeitet sowohl für Deliveroo als auch für Helpling. Um ihre Miete zu finanzieren, zieht sie gelegentlich zu ihrem Freund und vermietet ihre Wohnung unter der Hand für einige Tage über Airbnb: Leben unter Bedingungen des Plattform-Urbanismus.

Digitale Plattformen verändern die Welt der urbanen Arbeit umfassend. Das gilt nicht nur, aber in besonderer Weise für Migrant_innen. Deren Mobilität, Prekarisierung und Flexibilisierung bildet die Grundlage derartiger Arbeitsverhältnisse in der digitalen Ökonomie. Während die Arbeit auf der Putzplattform Helpling unsichtbar und in privaten Räumen bleibt, sind die Fahrer_innen der Essenslieferdienste oder die inzwischen mehrere Tausend Uber-Taxis aus dem Stadtbild Berlins kaum noch wegzudenken. Das lässt wiederum darauf schließen, dass sich mit der Transformation der Arbeit auch Praktiken und Muster urbanen Konsums, transnationaler Mobilität und sozialer Reproduktion verändern - und damit der urbane Raum selbst. Neben den bisher genannten Plattformen müssen noch viele weitere als Teil dieser Veränderung berücksichtigt werden: von Airbnb bis Google, von TripAdvisor bis Amazon. Deswegen wollen wir im Folgenden unseren Blick über die Plattformarbeit hinaus richten und diesen emergenten PlattformUrbanismus grob skizzieren.

\section{Plattform-Urbanismus: \\ Geisterküchen und On-Demand-Geografien}

Kehren wir zurück zu dem virtuellen Restaurant in Berlin-Kreuzberg im Juni 2019, von dem der Deliveroo-Kurier die Bestellung abgeholt hat. Es liegt versteckt im zweiten Hinterhof. Wer sich hier nach einem konventionellen Restaurant umschaut, wird lange suchen müssen. So wie der Kurier, als er zum ersten Mal hier eine Bestellung abholte. Neben einer unscheinbaren schwarzen Tür hängt ein dezentes Schild mit dem Firmennamen und den Logos gleich mehrerer in Berlin tätiger Essenslieferdienste. Zahlreiche Fahrradkuriere gehen ein und aus. Hinter der Tür öffnen sich die Räume zur Küche. In diesen 
virtuellen Restaurants ist alles für die Auslieferung optimiert. Die üblichen kulturellen Insignien eines Restaurants fehlen vollständig, alles findet unter der Maßgabe von Hygiene und Effizienz statt. An einem Regal hängen zahlreiche Tablets, über die im Minutentakt Bestellungen eingehen, die irgendwo in der Stadt per App getätigt wurden. Einige der beliebtesten „Restaurants“ auf diesen Plattformen existieren tatsächlich nur in diesem Raum. In diesem Geisterrestaurant werden mexikanische Burritos, Thaicurry, vegane Hotdogs und hawaiianische Poke Bowls direkt nebeneinander gekocht. Den meisten Kund_innen wird das nicht auffallen: Die Kochstile und Nationalküchen, die hier von denselben Köch_innen zubereitet werden, treten in den Apps der Lieferdienste wie verschiedene Restaurants auf.

Global entstehen in urbanen Kontexten immer mehr solcher Restaurants, in denen ausschließlich für die Lieferung gekocht wird. Oft werden sie als „dark kitchens“, „cloud kitchens“ oder „ghost kitchens“ bezeichnet. Weil die bestehenden Restaurants, die gleichzeitig Laufpublikum bedienen müssen, sich oft als zu ineffizient für die Anforderungen der Auslieferung erweisen und die beliebten Innenstadtlagen für solche Restaurants mit hohen Mietkosten verbunden sind, steigt die Zahl dieser Geisterrestaurants und damit die Bedeutung der sie begleitenden Geschäftsmodelle. So baute beispielsweise Travis Kalanick, der Mitgründer und ehemalige CEO von Uber, die Firma Cloud Kitchens auf, die auf die Auslieferung optimierte „virtuelle Küchen“ an bestehende Restaurants vermietet, damit sie diese von ihren Restaurantküchen trennen können. Kitchen United, ein von Google finanziertes Start-up, bietet neben Räumen und Küchenausstattung auch Software für Lieferküchen an und verspricht dabei eine Einsparung von 75 bis 80 Prozent des Personals im Vergleich zu herkömmlich organisierten gastronomischen Küchen.

Auch die Lieferplattform Deliveroo hat auf die Erfahrung reagiert, dass viele Restaurants mit der Nachfrage nach Essenslieferungen überfordert sind und das Programm „Deliveroo Editions“ aufgesetzt. So stehen beispielsweise auf einem heruntergekommenen Parkplatz im Londoner Stadtteil Blackwell, der einst ein Umschlagplatz für Kleidung und Wolle war, zehn fensterlose Container in den Farben der Plattform. In jedem dieser kleinen Container arbeitet das Küchenpersonal beliebter Restaurants aus der Umgebung und kocht Essen - ausschließlich für die Auslieferung, etwa in die benachbarten Bürotürme in Canary Wharf, einem früheren Teil des Hafens, der jetzt ein neues Finanzzentrum ist.

Die Container, die, abgesperrt durch Metallzäune unter den Schienen der Hochbahn stehen und mit Flutlicht beleuchtet werden, bieten ein eher trostloses Bild. Das Personal beschwert sich über die Temperaturen in den fensterlosen Containern: Je nach Jahreszeit ist es zu kalt oder zu heiß. In anderen Londoner Stadtteilen oder in anderen Städten Großbritanniens, Australiens, Frankreichs oder in Hongkong, in denen es solche Geisterküchen gibt, nutzt „Deliveroo Editions“ alte Warenhäuser oder Fabrikgebäude. Neben der großen Kapazität und den geringen Mieten sind effiziente Abläufe ein zentraler ökonomischer Faktor dieser „virtuellen Küchen“. Zur Projektierung solcher Editions-Küchen nutzt Deliveroo die Daten aus Millionen vorhergegangener Bestellungen. Mit diesen lasen sich unter anderem die Nachfrage nach bestimmten Restaurants und Kochstilen für potenzielle Standorte bestimmen. Die Editions-Küchen sowie die zunehmende Automatisierung und 
Standardisierung des Kochens spielen eine wichtige Rolle für das langfristige Unternehmensziel, die gelieferten Essen preislich an die Kosten für selbst gekochtes Essen anzunähern.

\section{Logistische Städte und neuer Plattform-Urbanismus}

Diese Entwicklungen zeigen, dass Plattformen wie Deliveroo Teil tiefgreifender Veränderungen sind, die nicht nur die Welt der Arbeit betreffen, sondern auch die Transformation des urbanen Raums. Produktion und Konsumption von Essen sind ein wichtiger Faktor, der den urbanen Raum, seine Kultur und seine Sozialität prägt, insbesondere in Innenstädten. Im Kontext einer sich immer mehr verbreitenden On-Demand-Logik verändert sich jedoch nicht nur das Essensverhalten, sondern auch das Konsumverhalten insgesamt. Immer mehr Waren - von Büchern über Käse zu Waschmaschinen - werden bis an die Haustüre geliefert. Die logistischen Prozesse bei der letzten Meile der Auslieferung werden dabei immer wichtiger; die Anforderungen an Geschwindigkeit, Flexibilität und Effizienz steigen. Amazon benötigt beispielsweise seit einigen Jahren in den Innenstädten neue Distributionszentren, um seine Prime-Kundschaft zu beliefern, die teilweise mit „Same Hour“-Lieferversprechen umworben wird. In Berlin entstanden neue städtische Amazon-Distributionszentren zuerst am Kurfürstendamm und später in Tegel. Diese neuen, innerstädtischen Lieferzentren tauchen jüngst in vielen größeren Städten auf. Sie ergänzen die großen Distributionszentren, die Amazon normalerweise an strukturschwachen und oftmals von hoher Arbeitslosigkeit gekennzeichneten Orten in der Peripherie von Städten platziert, um Miete und Lohnkosten zu sparen - wie in Brieselang bei Berlin.

In ihrem Buch Learning from Logistics spekuliert die Urbanistin und Architektin Clare Lyster, dass die Logistik inzwischen zum zentralen Paradigma der Stadtplanung geworden ist, da Ströme von Menschen, Waren und Daten eine immer größere Bedeutung bekommen. Um diesen neuen Urbanismus zu verstehen, schlägt Lyster vor, Zeit und Effizienz als die wichtigsten Vektoren der Urbanisierung zu begreifen und fordert uns auf: „Hypothesize that time is the most critical attribute of city making, reconceptualise the city as integrated service platform rather than a series of figural artefacts.“ (Lyster 2016: 13) Im Blick auf die logistischen Geografien der On-Demand-Logik, die Same-Day- oder Same-Hour-Versprechen der Plattformen, die variablen Lieferzonen, die Stadtbewohner_innen bestimmte Dienstleistungen ermöglichen oder sie von ihnen ausschließen oder die Heat Maps, die Nachfrage in Echtzeit darstellen und automatisch mit Anreizsystemen für Taxi- oder Lieferfahrer_innen verbinden, lassen sich die Umrisse dieser Rationalität erkennen (vgl. Altenried 2019).

Die neuen Distributionszentren, die Geisterküchen und die von Fahrradkurier_innen und Liefervans bevölkerten Straßen sind sichtbare Ausdrücke materieller Veränderungen urbanen Lebens. Dazu kommen noch viele weitere, zum Teil weniger sichtbare Plattformen. Man denke an die versteckten Geografien urbaner Reproduktionsarbeit und deren Re-Organisation über Plattformen wie Helpling oder Care.com (Altenried/Dück/Wallis 2021), an WeWork - ein Start-up, das Co-Working-Spaces und Büroflächen kurzfristig 
vermietet - oder an die omnipräsente Ferienwohnungsplattform Airbnb mit ihren teils drastischen Auswirkungen auf Immobilienmärkte, Praktiken des Wohnens und Vermietens sowie auf Gentrifizierungsprozesse. Ein weiteres Beispiel sind Uber, ShareNow, Lime und ihre Effekte auf urbane Mobilität. Manchmal scheint es, als gäbe es keinen Sektor urbanen Lebens, der sich nicht „uberisieren“ ließe. All diese Plattformen interagieren mit den vorhandenen städtischen Ökonomien und Infrastrukturen und transformieren diese zum Teil drastisch. Plattformen spielen auch eine wichtige Rolle beim Aufstieg der sogenannten Smart City. Der Begriff bezeichnet ein Ensemble aus Technogien, Diskursen und neuen Formen der Inwertsetzung des Urbanen, in dem große Plattformen und Technologieunternehmen eine wichtige Rolle spielen.

Wie lassen sich diese komplexen Entwicklungen analysieren und konzeptualisieren? Hier kommt der Begriff des Plattform-Urbanismus ins Spiel, wie das gleichnamige Buch von Sarah Barns (2020), der digitale Roundtable der Zeitschrift Mediapolis (Rodgers/Moore 2018) oder der Schwerpunkt der Zeitschrift Urban Geography (Sadowski 2020) zeigen. Weder gemeinsam noch einzeln liefern diese Debattenbeiträge einen kohärenten Theorieansatz oder einen fertigen analytischen Rahmen. Sie bieten aber Ansatzpunkte für ein Nachdenken über die neueren Entwicklungen und die Urbanisierung des digitalen Kapitalismus. Der Begriff des Plattform-Urbanismus erlaubt einerseits eine Analyse der Verräumlichung von Plattformen und der Geografien des Plattform-Kapitalismus (Srnicek 2016). Der Blick auf die Geografie von Plattformen zeigt andererseits, dass diese meist ein dezidiert urbanes Phänomen sind.[3] Uber, Deliveroo oder Helpling können ihre Netzwerkeffekte am besten im verdichteten Raum der Stadt entfalten. Hier, wo sie auf mobile, prekäre und flexibilisierte Arbeit zurückgreifen können, entwickeln sie ihre Dynamik.

Wie bereits erwähnt wollen wir die unterschiedlichen Plattformen und ihre variablen Einflüsse auf städtisches Leben nicht vereinheitlichen. Zugleich fehlt es hier am nötigen Platz, um die Umrisse einer Theorie des PlattformUrbanismus vertieft zu diskutieren. Dennoch erscheint uns die Infrastrukturwerdung von Plattformen als möglicher Ausgangspunkt für solche Überlegungen zentral zu sein. Wie wir gezeigt haben, zielen die meisten Plattformen auf unterschiedliche Weisen darauf ab, unverzichtbare Infrastrukturen des Alltagslebens zu werden. Die beobachtbare Plattformisierung von Infrastruktur und die Infrastrukturisierung von Plattformen (vgl. Plantin et al. 2018) lassen sich dementsprechend vielleicht als die zentrale Wirkungsweise von Plattformen auf den urbanen Raum fassen. Die Diskussion des PlattformUrbanismus im Kontext der digitalen Re-Konfiguration räumlicher und informationeller Infrastruktur (Easterling 2014, Bratton 2016) erschließt auch eine politische Kritik der plattformgetriebenen Transformation des urbanen Raums. Schließlich zeichnet sich die Plattformisierung städtischer Infrastruktur fast immer durch eine massive Ökonomisierung dieser Infrastrukturen aus. Zudem geht sie mit einer Verschärfung existierender Ungleichheiten und der immer weitergehenden Inwertsetzung neuer Bereiche urbanen Lebens einher. Das aggressive Vorgehen von Plattformen wie Uber und Airbnb sowie die Probleme verschiedener Städte, sich dagegen zu wehren, markieren die Relevanz dieses Phänomens. Die Arbeitskämpfe auf verschiedenen Plattformen oder der breite Widerstand gegen Airbnb mit seinen globalen 
Auswirkungen zeigen allerdings auch, dass der Prozess der Plattformisierung der Stadt nicht linear und unwidersprochen verläuft. Auch die Algorithmen der Arbeitsplattformen sind nicht allmächtig, Arbeiter_innen finden immer wieder Lücken und Praxen, um die Algorithmen zu ihrem Vorteil auszutricksen. Obwohl die Plattformen selbst gerne den Anschein erwecken, ist die Plattformisierung keineswegs ein lückenloser, unumkehrbarer und allumfassender Prozess, sondern praktisch auf jeder Ebene umkämpft und kontingent.

\section{Schluss: Berlin, März 2020}

Im Vergleich zur eingangs beschriebenen Szene hat sich viel verändert. Nur wenige Wochen später, im August 2019, hat die Plattform Deliveroo ihr Deutschlandgeschäft eingestellt. Über die Gründe lässt sich nur spekulieren. Die circa 1.00o Berliner Fahrer_innen der Plattform waren davon völlig überrascht. Wenige Tage bevor die Entscheidung öffentlich bekannt wurde, hatten alle Deliveroo-Fahrer_innen eine Nachricht erhalten, dass im Büro neue Ausrüstung für sie zur Abholung bereitliege. Kurz darauf folgte eine E-Mail, die das Ende der Plattform zum Ende der Woche ankündigte. Der spontane Rückzug löste bei vielen Unglauben und Panik aus, bedeutete er doch für sie plötzliche (Teil-)Arbeitslosigkeit und massive finanzielle Einbußen. Er verdeutlichte so auf drastische Weise die enorme Prekarität der Plattformarbeit. Die Einstellung verweist darauf, dass die meist risikokapitalfinanzierten Interventionen von Plattformen in den urbanen Raum auch im Falle ihres Scheiterns drastische Folgen haben können.

Wenige Monate später, im März 2020, arbeiten viele der ehemaligen Deliveroo-Fahrer_innen beim Konkurrenten Lieferando, der inzwischen das Geschäft mit Essenslieferungen in Berlin dominiert. Ihr Alltag hat sich damit jedoch nicht wieder normalisiert. In Folge der Covid-19-Pandemie ist die Stadt im Lockdown. Obwohl Lieferando seit Beginn der Krise mit „kontaktloser Lieferung"wirbt, sind die Fahrer_innen erheblichen gesundheitlichen Risiken ausgesetzt. Sie schwanken zwischen der Angst, sich durch die zahlreichen Kontakte mit der Kundschaft und in Restaurants anzustecken und der Befürchtung, dass ihre Tätigkeit eingestellt wird. Lieferando versprach zwar Schutzausrüstung, lieferte diese aber zur Verärgerung vieler Fahrer_innen wochenlang nicht aus. Im Gegensatz zu Deliveroo sind die Fahrer_innen bei Lieferando per Minijobs oder mit anderen Teilzeitverträgen angestellt. So stehen sie bei der Befürchtung sich anzustecken immerhin vor der Wahl, Urlaub zu nehmen oder Fehlzeiten anzuhäufen. Solo-selbstständige Arbeiter_innen anderer Plattformen haben keine derartige Absicherung. Arbeiter_innen von Helpling berichten, dass die Aufträge seltener werden und sie ohne Schutzausrüstung Angst haben, die Wohnungen der Kund_innen zu betreten. Wie unzählige Plattformarbeiter_innen auf der ganzen Welt stehen sie derzeit vor der Wahl, entweder auf Einkommen zu verzichten oder ihre Gesundheit aufs Spiel zu setzen. Hier zeigt sich besonders deutlich das stratifizierte Risiko in Zeiten der globalen Pandemie. Für mobile Bevölkerungen gilt dies sowohl hinsichtlich ihrer Arbeit als auch ihres prekären Zugangs zur Gesundheitsversorgung.

Auch wenn einige Plattformen wie Airbnb und Uber während der Covid19-Pandemie zunächst deutlich unter dem generellen Rückgang der Mobilität 
litten, spricht vieles dafür, dass die Pandemie auflängere Sicht zum weiteren Aufstieg der Plattformen beitragen wird. Während der ersten Lockdowns etwa in Paris und Mailand waren die Fahrer_innen von Deliveroo und anderen Lieferdiensten oft die einzigen Menschen, die noch auf den leer gefegten Straßen zu sehen waren. Im Vereinigten Königreich führten Uber, Deliveroo und Just Eat Gespräche mit der Regierung, um gegebenenfalls die Versorgung älterer Menschen zu übernehmen. Seit Beginn der Covid-19Krise bieten viele dieser Essensplattformen zusätzlich die Lieferung von Gütern des täglichen Bedarfs an. In China führte der Ausbruch von Covid-19 zu einem Boom von Plattformen, die Essen und Lebensmittel an die Haustüren der isolierten Kund_innenschaft liefern. Auch in Deutschland ist die Nachfrage nach Lieferungen ins eigene Zuhause enorm gestiegen. Amazon stellte Hunderttausende neue Liefer- und Lagerarbeiter_innen ein, um die explodierende Nachfrage zu bewältigen. Jetzt, im Zuge der Covid-19-Krise, zeigt sich die gesellschaftliche Relevanz dieser Plattformen noch deutlicher, ebenso wie die Prekarität ihrer Arbeitsmodelle: Sie sind ein Gesundheitsrisiko für vorwiegend migrantische Arbeitskräfte, die sich damit in diesen Zeiten ihre Reproduktion sichern. Daher nimmt zugleich auch die Kritik an diesem Geschäftsmodell zu. Einige Plattformen sehen sich inzwischen genötigt, ihre Arbeiter_innen im Krankheitsfall zu unterstützen. Auch wenn diese Unterstützung Garantien und soziale Rechte nicht ersetzen können, sind sie doch Ausdruck eines politischen Drucks nicht zuletzt der Plattformarbeiter_innen selbst. Auch dieser verstärkt sich mit der aktuellen Krise weiter.

Dieser Artikel wurde durch Publikationsmittel im Forschungsprojekt Plattform Labour in Urban Spaces (PLUS) gefördert, finanziert durch die Europäische Kommission im Rahmen des Programms Horizon 2020 (Grant agreement No. 822638).

\section{Endnoten}

[1] Eshandelt sich zum einen um das von der Deutschen Forschungsgemeinschaft(DFG) unterstützte Projekt „Digitalisierung von Arbeit und Migration“ (Fördernummer 398798988), in dem wir (Moritz Altenried und Manuela Bojadžijev) gemeinsam mit Mira Wallis und Felix Busch-Geertsema forschen: http://www.platform-mobilities.net/. Zum anderen geht es um das von der Europäischen Kommission im Rahmen des Programms Horizon 2020 geförderte Forschungsprojekt „Platform Labour in Urban Spaces“ (PLUS, Grant agreement No. 822638), in dem außer uns (Moritz Altenried, Stefania Animento und Manuela Bojadžijev) Valentin Niebler und Roxana Weger arbeiten. Die Unterstützung der Europäischen Kommission für die Erstellung dieser Veröffentlichung stellt keine Billigung des Inhalts dar, welcher nur die Ansichten der Verfasser wiedergibt, und die Kommission kann nicht für eine etwaige Verwendung der darin enthaltenen Informationen haftbar gemacht werden.

[2] Sämtliche Namen von Interviewten haben wir geändert.

[3] Eine Ausnahme bilden sogenannte Crowdworking-Plattformen, deren genuine Qualität darin besteht, über den gesamten Globus verteilte digitale Arbeiter_innen miteinander zu verbinden. Crowdworking-Plattformen bilden eine Art digitaler Fabrik räumlich verteilter Heimarbeiter_innen und kreieren so eine eigenständige Geografie, die sich nicht unbedingt mit den urbanen Zentren deckt. 


\section{Autor_innen}

Stefania Animento hat ihre Schwerpunkte im Bereich Mobilität, Migration und Klasse sowie Stadt- und Gentrifizierungsforschung.

animento@leuphana.de

Manuela Bojadžijev interessiert sich für gegenwärtige Transformationsprozesse von Mobilität und Migration sowie von Rassismus, im Zusammenspiel mit Veränderungen von Arbeit und Alltag durch Digitalisierung und Logistik, vorwiegend in urbanen Räumen.

manuela.bojadzijev@hu-berlin.de

Moritz Altenried arbeitet empirisch und theoretisch unter anderem zu Digitalisierung, Arbeit, Migration, Plattformen und Logistik im globalen Kapitalismus.

moritz.altenried@hu-berlin.de

\section{Literatur}

Altenried, Moritz (2019): On the last mile: Logistical urbanism and the transformation of labour. In: Work Organisation, Labour \& Globalisation 13/1, 114-129.

Altenried, Moritz (i. E.): The digital factory. The human labor of automation. Chicago/ London: University of Chicago Press.

Altenried, Moritz / Bojadžijev, Manuela / Höfler, Leif Jannis / Wallis, Mira / Mezzadra, Sandro (2017): Logistische Grenzlandschaften. Das Regime mobiler Arbeit nach dem Sommer der Migration. Münster: Unrast.

Altenried, Moritz / Dück, Julia / Wallis, Mira (Hg.) (2021): Plattformkapitalismus und die Krise der sozialen Reproduktion. Münster: Westfälisches Dampfboot.

Animento, Stefania / Di Cesare, Giorgio / Sica, Cristian (2017): Total eclipse of work? In: PROKLA. Zeitschrift für kritische Sozialwissenschaft 47/2, 271-290.

Barns, Sarah (2018): Smart cities and urban data platforms. Designing interfaces for smart governance. In: City, culture and society 12, 5-12.

Barns, Sarah (2020): Platform urbanism. Negotiating platform ecosystems in connected cities. Singapore: Palgrave Macmillan.

Beverungen, Armin (2017): Algorithmisches Management. In: Timon Beyes / Jörg Metelmann / Claus Pias (Hg.), Nach der Revolution. Ein Brevier digitaler Kulturen. Berlin: Tempus, 51-63.

Beverungen, Armin / Sprenger, Florian (2017): Editorial: Computing the City. In: The Fibreculture Journal 29. http://twentynine.fibreculturejournal.org/issue-29-fcj-212-computing-the-city/ (letzter Zugriff am 3.10.2020).

Bojadžijev, Manuela (i. E.): “The spirit of Europe”. Differential migration, labour and logistification. In: Sandro Mezzadra / Giorgio Grappi (Hg.), Contested justice. Europe and migration in a global perspective. London: Routledge.

Bratton, Benjamin H. (2016): The stack. On software and sovereignty. Cambridge (Mass.): MIT Press.

Briken, Kendra (2018): Arbeit. In: Bernd Belina / Matthias Naumann / Anke Strüver (Hg.), Handbuch kritische Stadtgeographie. 3. Auflage. Münster: Westfälisches Dampfboot, 241-246.

Cant, Callum (2019): Riding for Deliveroo. Resistance in the new economy. London: John Wiley \& Sons.

Easterling, Keller (2014): Extrastatecraft: The power of infrastructure space. London: Verso. Gillespie, Tarleton (2010): The politics of "platforms". In: New Media \& Society 12/3, 347-364.

Halpern, Orit / LeCavalier, Jesse / Calvillo, Nerea / Pietsch, Wolfgang (2013): Test-bed urbanism. In: Public Culture 25/270, 272-306.

Harvey, David (2010): Social justice and the city. Athens/London: Univ. of Georgia Press.

Harvey, David (2013): Rebellische Städte. Berlin: Suhrkamp.

Heeks, Richard (2019): How many platform workers are there in the global south? ICTs for development. In: ICT4DBlog, 29.1.2019. https://ict4dblog.wordpress.com/2019/01/29/ how-many-platform-workers-are-there-in-the-global-south/ (letzter Zugriff am 22.4.2020). 
Huws, Ursula / Spencer, Neil / Coates, Matthew / Sverre Syrdal, Dag / Holts, Kaire (2019): The platformisation of work in Europe. Results from research in 13 European countries. Brussels: Foundation for European Progressive Studies (FEPS).

Luque-Ayala, Andrés / Neves Maia, Flávia (2019): Digital territories. Google maps as a political technique in the re-making of urban informality. In: Environment and Planning D: Society and Space 37/3, 449-467.

Lyster, Claire (2016): Learning from logistics. How networks change our cities. Basel: Birkhäuser.

Miles, Sam (2017): Sex in the digital city. Location-based dating apps and queer urban life. In: Gender, Place \& Culture 24/11, 1595-1610.

Pesole, Annarosa / Brancati, Cesira Urzi / Fernández-Macías, Enrique / Biagi, Federico / González Vázquez, Ignacio (2018): Platform workers in Europe. Luxembourg: Publications Office of the European Union.

Plantin, Jean-Christophe / Lagoze, Carl / Edwards, Paul N. / Sandvig, Christian (2018): Infrastructure studies meet platform studies in the age of Google and Facebook. In: New Media \& Society 20/1, 293-310.

Rodgers, Scott / Moore, Susan (2018): Platform urbanism. An introduction. In: Mediapolis 23/4, https://www.mediapolisjournal.com/2018/10/platform-urbanism-an-introduction/ (letzter Zugriff am 22.4.2020).

Rosenblat, Alex (2018): Uberland. How algorithms are rewriting the rules of work. Oakland: University of California Press.

Sadowski, Jathan (2020): Cyberspace and Cityscapes. On the emergence of platform urbanism. In: Urban Geography 41/3, 1-5.

Srnicek, Nick (2016): Platform capitalism. Cambridge: Polity.

Wachsmuth, David / Weisler, Alexander (2018): Airbnb and the rent gap. Gentrification through the sharing economy. In: Environment and Planning A: Economy and Space 50/6, 1147-1170.

Wills, Jane / Datta, Kavita / Evans, Yara / Herbert, Joanna / May, Jon / McIlwaine, Cathy (2010): Global cities at work. New migrant divisions of labour. London/New York: Pluto Press.

Woodcock, Jamie / Graham, Mark (2019): The gig economy. A critical introduction. Cambridge: Polity.

Xiang, Biao / Lindquist, Johan (2014): Migration infrastructure. In: International Migration Review 48, 122-148.

\section{Platform urbanism. Labour, migration and the transforma- tion of urban space}

The contribution analyses how digital platforms transform labour and life just as well lived space and material architecture of the contemporary city. This concerns not only labour relations, but also everyday forms and practices of mobility, consumption or reproduction. Based on extensive ethnographic research, we describe, firstly, the rise of platform labour in Berlin, with a focus on Uber, Deliveroo and Helpling. We analyse new forms of algorithmic organization, control and measure of labour in urban space and describe platform labour as primarily migrant work. Secondly, we sketch the outlines of an emerging platform urbanism, which includes an understanding of the spaces and geographies of digital platforms as well as a theoretical perspectivation of the term. And thirdly, we emphasize that critical analyses of emerging platform urbanism can help to understand how platforms aim to become indispensable urban infrastructures. This infrastructural emergence of urban platforms is not a smooth process, however, but is politically and economically contested. 